\title{
Concentrated Photovoltaic Thermoelectric Hybrid System: An Experimental and Machine Learning Study
}

\author{
Zeming He, ${ }^{1,2, \#}$ Ming Yang, ${ }^{1,2, \#}$ Lei Wang, ${ }^{3}$ Ergude Bao ${ }^{3, *}$ and Hang Zhang ${ }^{1,2, *}$
}

\begin{abstract}
Applying solar energy over a wider spectral range can lead to more efficient energy conversion. The combination of a photovoltaic (PV) cell and a thermoelectric generator (TEG) is a widely studied technology for effectively broadening the use of the solar spectrum. In this paper, we select two kinds of photovoltaic cells and combine them with a TEG to form different systems, and analyze the overall performance of each system to provide a certain reference for optimal use of photovoltaic cells and a TEG in a hybrid system. Furthermore, we use machine learning to optimize the structural parameters of the hybrid system, and predict the optimal output power of the system when the area ratio of the TEG and PV module is 4.41. This work provides an important reference for further research on the PV-TEG hybrid system and its applications.
\end{abstract}

Keywords: PV-TEG hybrid system; Performance analysis; Machine learning.

Received: 25 February 2021; Accepted date: 17 March 2021.

Article type: Research article.

\section{Introduction}

The demand for renewable energy and waste heat recovery is increasing as fossil energy consumption and the need for environmental protection grow. To deal with this situation, it is necessary to develop an environmentally-friendly energy system by taking advantage of renewable energy. The energy from the sun is considered to be renewable and sustainable. ${ }^{[1-3]}$ Solar energy has been used to generate electricity via solar cells for many years. Photovoltaic energy has some advantages such as inexhaustibility, which helps reduce pollution and conserves fossil resources, but the use of solar energy is limited because it is difficult to fully use the solar spectrum. The part of solar energy that cannot be used by photovoltaic cells, such as infrared energy, is converted into waste heat. ${ }^{[4-6]}$ Although the output power and efficiency of photovoltaic (PV) cells are enhanced using optical concentrators to increase the intensity of solar radiation, ${ }^{[7-9]}$ the $\mathrm{PV}$ cell temperature increases significantly

\footnotetext{
${ }^{1}$ Institute of Engineering Thermophysics, Chinese Academy of

Sciences, Beijing, China.

${ }^{2}$ University of Chinese Academy of Sciences, Beijing 100049,

China.

3 School of Software Engineering, Beijing Jiaotong University, Beijing 100044, China.

\#These authors contributed to this work equally.

*E-mail: zhanghang@iet.cn (H. Zhang), baoe@bjtu.edu.cn (E. Bao)
}

in a concentrated PV (CPV) system, which reduces efficiency. Therefore, the excess thermal energy should be removed to overcome this drawback of a concentrated PV system. ${ }^{[10]}$

Recently, much attention has been given to a PV system with a thermoelectric generator (TEG) in which the PV cell is cooled by converting part of its heat into electricity via the Seebeck effect using thermoelectric materials. ${ }^{[11-15]}$ The incident solar flux on the PV cell or thermoelectric generator can be adjusted by selecting from several collectors such as Fresnel lenses, parabolic troughs, and parabolic dishes. ${ }^{[16,17]}$ In this PV-TEG hybrid system, the TEG is directly connected to the PV cell to recover the waste heat and improve the conversion efficiency.

The theory of PV-TEG hybrid systems has been extensively developed. Meanwhile, studies have been done on enhancing the conversion efficiency of a concentrated PV-TEG system and on its practical feasibility. ${ }^{[18]}$ Van Sark presented a simple model of a PV-TEG hybrid system in which the TEG is connected to the back of the PV cell to harvest and use its thermal waste, and theoretically found that this enhanced the efficiency of the system by $8-23 \%{ }^{[19]}$ Lamba and Kaushik presented a theoretical model of a CPV-TEG hybrid system based on the first and second laws of thermodynamics and analyzed the influence of different parameters like concentration ratio, solar irradiance, and the number of TEG thermoelements on system performance. The 
results showed that the efficiency of the hybrid PV-TEG system was $13.37 \%$ more than that of a PV-only system for a concentration ratio of 3 with 127 thermoelements. ${ }^{[20]}$

Mahmoudinezhad et al. developed a numerical model for the transient response of a CPV-TEG hybrid system, and found that the efficiency of the PV decreased and that of the TEG increased with an enhanced concentration ratio. The results showed that using a TEG in such a hybrid system yielded a more stable output power. ${ }^{[21,22]}$ Rezania and Rosendahl developed a thermally coupled model to predict the performance of a CPV-TEG hybrid system. Critical parameters were considered to evaluate the economic viability of the hybrid system compared with that of commercial CPV systems. The results showed that the efficiency of the hybrid system was higher than that of a CPV-only system, and that the TEG significantly impacted power generation by the hybrid system, especially at high solar concentrations. ${ }^{[23]} \mathrm{Wu}$ et al. analyzed and compared the performance of a stand-alone PV system, solar PV-TEG hybrid system, and solar PV-TE cooling hybrid system and found the PV-TEG hybrid system had increased total output power and the PV-TE cooling system had reduced PV cell temperature. ${ }^{[24]}$ Kraemer et al. developed and numerically analyzed a spectrum-splitting PV-TEG hybrid system and compared the efficiencies of hybrid systems with crystalline silicon, amorphous silicon, and heterojunction thin-film PV modules for different solar TEGs. The results provided useful information for partitioning the solar spectrum to yield maximum conversion efficiency of a PV-TE hybrid system. ${ }^{25}$ Zhu et al. presented a thermal CPV-TEG hybrid system with optimized thermal management and theoretically calculated its heat flow and temperature distribution. The hybrid system used a copper plate serving as a thermal concentrator and conductor to maintain a large temperature difference on both sides of the TEG, which made the hybrid system achieve a high peak efficiency of $23 \%$ in an outdoor test. ${ }^{[26]}$ Liao et al. presented and discussed the performance characteristics of a device consisting of a weakly concentrated PV module and a TEG, calculated its maximum power output, and determined the optimal electric resistances of the CPV and TEG. They found optimum values for the thermal conductance between

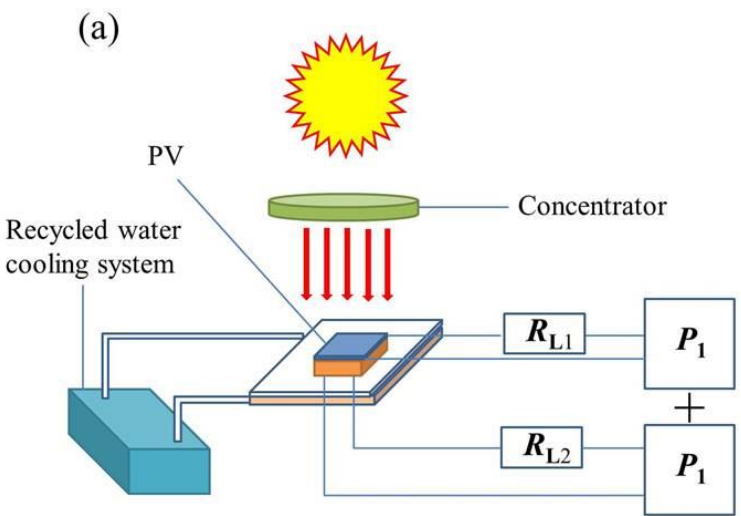

the CPV and the TEG, the CPV current, the solar irradiation, the concentration ratio, and other important parameters. ${ }^{[2]]}$

Machine learning is a technology in which a computer learns from existing knowledge and experience to model data to predict future results or trends. Deep learning is a special machine learning that needs a lot of data support. It involves making decisions according to input and output data by imitating the perception and organization of the human brain. Deep learning has developed rapidly in recent years, especially after a team led by Hinton, the "Father of Neural Networks," won the first prize for its deep learning algorithm in the 2012 ImageNet Large-Scale Visual Recognition Challenge (ILSVRC). As a result, deep learning has gradually become the main focus of research in natural language processing, computer vision, and other fields. Because deep learning can save experimental time, money, and manpower, physics research has gradually begun to use deep learning methods such as deep neural networks to achieve new physical signals and improve background selection, ${ }^{[28]}$ and deep learning algorithms to predict crystal enthalpy of formation. ${ }^{[29]}$ Deep learning has also been applied to gas turbines, particulate matter, organic Rankine cycles, and hydrogen array sensor detection. ${ }^{[30]}$

However, most works on CPV-TEG hybrid performance and optimization focused on theoretical studies rather than experimental studies. Machine learning plays an important role in research on energy systems and devices. Hence, in this paper, we establish a hybrid system consisting of a low concentrator, a PV module, and a TEG that can use the waste heat from the CPV module. We analyze the parameter design and use machine learning to optimize the system.

\section{Experimental and machine learning methods 2.1 Experimental}

The schematic of the proposed CPV-TEG hybrid system is shown in Fig. 1. The system mainly consisted of a concentrator, a PV module, a TEG, and a water-cooling system. The TEG modules were made of bismuth telluride $\left(\mathrm{Bi}_{2} \mathrm{Te}_{3}\right)$. The PV modules and the TEG were placed in a parallel arrangement.

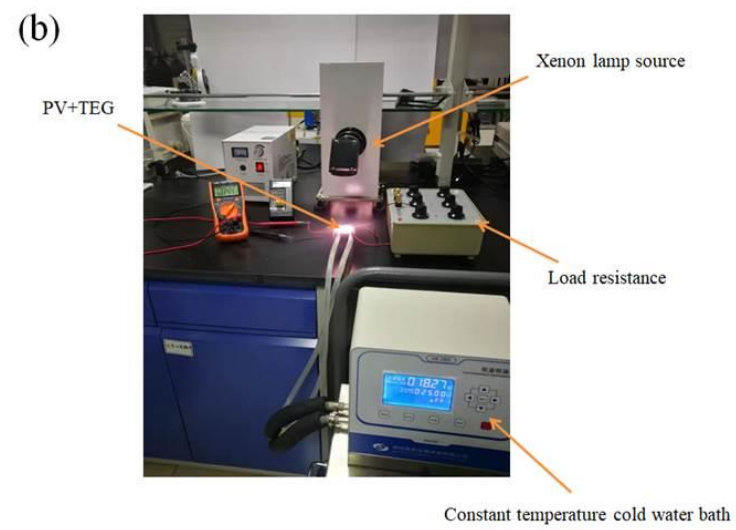

Fig. 1 CPV-TE hybrid system: (a) schematic; (b) photograph. 

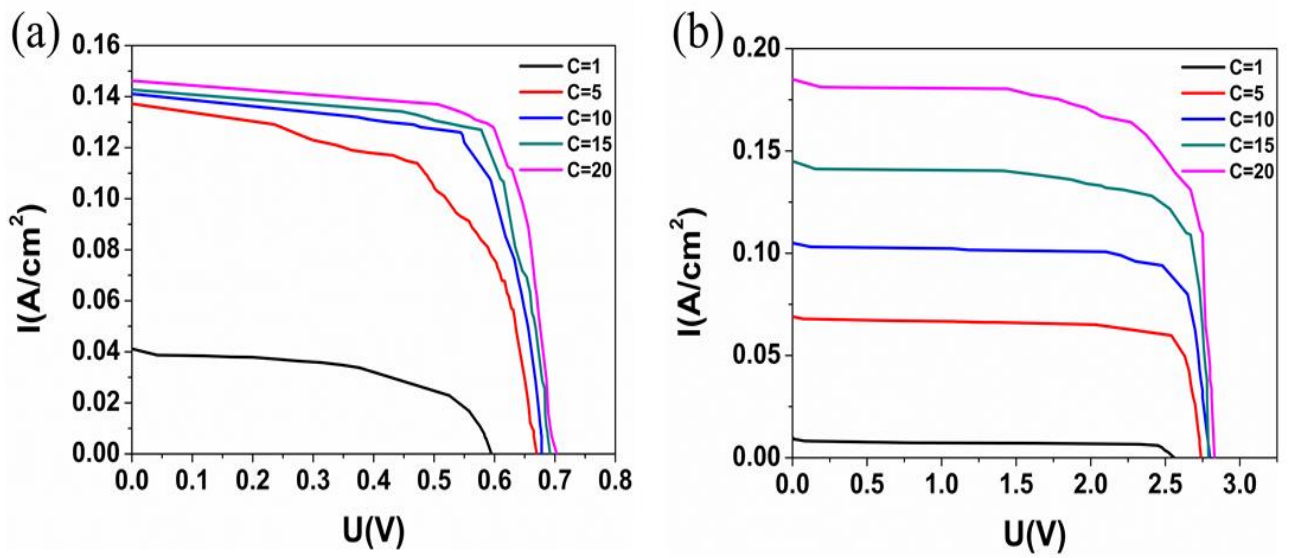

Fig. 2 Characteristic curves of PV modules at different concentration ratios at $\mathrm{G}=1000 \mathrm{~W} / \mathrm{m}^{2}$ : (a) mono-crystalline silicon; (b) gallium arsenide.

These PV modules mainly absorb shorter wavelengths (visible and UV regions), and the TEG absorbs longer wavelengths (IR region). The PV module is usually configured as the upper component and the TEG as the lower component, as shown in Fig. 1. The cooling system is placed at the back of the TEG to keep the temperature low on the cold side. Under sunlight, the PV module absorbs UV and visible light, while the rest of the radiation is transmitted to the hot side of the TEG through the PV module. The infrared radiation heats the top of the TEG, causing a temperature difference with the cold side. In the hybrid system, the PV and TEG modules are electrically isolated but thermally connected. Such a hybrid system makes the solar spectral energy available for use, thus improving the performance of the system. In the following, we will analyze the performances of the two components and the influence of their size ratio, and then investigate the performance of the hybrid system.

The experiments were conducted under indoor simulated light conditions to obtain steady results. A sun simulator was used as a light source to provide and concentrate sunlight; this consisted of a xenon lamp, reflector, and concentrator. The light intensity could be adjusted by changing the working power of the xenon lamp. The hybrid system was placed under the lamp, and the cooling temperature was controlled with the water-cooling system. The connecting interfaces between the PV, TEG, and cooling block were covered with a thin layer of thermal grease to reduce the thermal contact resistance.

The total input solar power, temperature, and output power of the system were measured during the experiment. A high-light power meter was used to measure the total input power of the system. The temperature was measured with K-type thermocouples. In a hybrid system, connecting the PV module and TEG in series or parallel may cause the total output power to decrease owing to the mismatch of the output characteristics between the PV and TEG. ${ }^{[31,32]}$ Thus, the PV and TEG worked separately and were measured with two electronic loads. The output power of the PV or TEG was obtained from the I-V curve measured with the electronic loads. Two types of solar cells, mono-crystalline silicon and gallium arsenide PV cells, were used in the experiment. Both were purchased commercially and not encapsulated to ensure better contact with the TEG to achieve better test results. The sizes of the PV and TEG are shown in Table 1.

Table 1. Device sizes.

\begin{tabular}{cccc}
\hline Items & Size of PV & Size of TEG & n value \\
\hline A & APV & ATEG & $\begin{array}{c}\text { ATEG } \\
\text { /APV }\end{array}$ \\
\cline { 2 - 4 } $\begin{array}{c}\text { represented } \\
\text { as the }\end{array}$ & $10 \mathrm{~mm} \times 10 \mathrm{~mm}$ & $10 \mathrm{~mm} \times 10 \mathrm{~mm}$ & 1 \\
cross-section & $10 \mathrm{~mm} \times 10 \mathrm{~mm}$ & $20 \mathrm{~mm} \times 20 \mathrm{~mm}$ & 4 \\
area of TEG & $10 \mathrm{~mm} \times 10 \mathrm{~mm}$ & $30 \mathrm{~mm} \times 30 \mathrm{~mm}$ & 9 \\
or PV & $10 \mathrm{~mm} \times 10 \mathrm{~mm}$ & $40 \mathrm{~mm} \times 40 \mathrm{~mm}$ & 16 \\
\hline
\end{tabular}

The mono-crystalline silicon and gallium arsenide PV cells were selected to compare performances of different photovoltaic cells. The characteristic curve of each PV cell is shown in Fig. 2. The curves show that the maximum powers of both PV cells increase with increasing concentration ratio. The gallium arsenide cell shows a higher power output than that of the mono-crystalline silicon, especially under a high concentration ratio. The TEG modules consisted of semiconductor elements and ceramic materials with high thermal conductivity that were connected electrically in series and thermally in parallel, and operated under steady conditions with negligible radiative and convective heat transfer from the sides of the TEG modules. The commercial $\mathrm{Bi}_{2} \mathrm{Te}_{3}$ TEG modules had sizes of $10 \times 10,20 \times 20,30 \times 30$, and $40 \times 40 \mathrm{~mm}^{2}$. The TEG modules differed mainly in size, while the amount of internal semiconductor materials, Seebeck coefficient, and thermal conductivity of the semiconductor materials were the same in all selected TEGs. In other words, the types, sizes, and properties of semiconductor materials were the same in all TEGs, and the 
differences in TEG sizes were only due to the differences in the number of thermoelectric pairs. This ensured that the experimental results would not be affected by differences in other properties of the semiconductor materials.

The dimensions of the PV module were $10 \times 10 \mathrm{~mm}^{2}$, and we assumed that $n=A_{T E G} / A_{P V}=1,4,9$ and 16 for the respective TEG sizes listed earlier, where $A$ is the cross-sectional area of the TEG or PV. We investigated the performance of the hybrid system for each value of $n$. The area of the TEG is larger than that of the PV cell when $n$ is greater than 1. For the experiment, we set up an insulation layer on the surface of the TEG to ensure that the heat received by the hot side of the TEG was only from the back of the PV module and not from the sunlight or other source.

\subsection{Power output and efficiency of the hybrid system}

The output power of the PV module is given by

$$
P_{P V}=I_{P V} \cdot V_{P V}
$$

where $I_{P V}$ and $V_{P V}$ are the output current and output voltage of the PV module, respectively.

The output power of the TEG is calculated from the measured data via

$$
P_{T E G}=I_{T E G} \cdot V_{T E G},
$$

where $I_{T E G}$ and $V_{T E G}$ are the output current and output voltage of the TEG module, respectively.

The electrical efficiency of the PV can be expressed as

$$
\eta_{P V}=\frac{P_{P V}}{A_{P V} \times C G}
$$

where $A_{P V}$ is the cross-sectional area of the PV module, $C$ is the concentration ratio, and $G$ is the irradiation intensity equal to $1000 \mathrm{~W} / \mathrm{m}^{2}$. The product $C G$ gives the solar radiation.

The conversion efficiency of the TEG can be evaluated as $^{[33]}$

$$
\eta_{T E G}=\eta_{c} \frac{M-1}{M+\left(\frac{T_{c}}{T_{h}}\right)} \eta_{o}=\eta_{P V}+\eta_{T E G},
$$

where $M=\sqrt{1+Z T_{m}}$ with $T_{m}=0.5\left(T_{h}+T_{c}\right) ; T_{h}$ and $T_{c}$ are the temperatures of the hot and cold sides of the TEG module, respectively; $T_{m}$ is the average temperature; $Z$ is the figure of merit of the TEG material, equal to $3.167 \times 10^{-3} 1 / \mathrm{K} ;{ }^{[34]} \eta_{c}$ is the Carnot efficiency, which is given by $\eta_{c}=\left(T_{h}-T_{c}\right) / T_{h}$.

The overall efficiency of the PV-TEG hybrid system can be express as

$$
\eta_{o}=\eta_{P V}+\eta_{T E G},
$$

and the output power of the PV-TEG hybrid system as

$$
P_{o}=P_{P V}+P_{T E G} \text {. }
$$

\subsection{Machine learning method}

We used a deep neural network (DNN) and a long short-term memory network without and with an attention mechanism (LSTM and LSTMA, respectively) to make a prediction. The power was predicted by inputting the external load, concentration ratio, cold-end temperature, material, and size to each model. All the models were trained with 80 epochs, a batch size of 1 , and a learning rate of $10^{-3}$. This was implemented in the framework PyTorch. Below are the detailed descriptions of the models.

\subsubsection{DNN model}

The neural network in the DNN includes the input layer, the output layer, and a large number of hidden layers. The layers are fully connected; that is, every neuron in the n-th layer is connected with every neuron in the $(n+1)$-th layer. For each layer, the DNN obtains the intermediate result $\mathrm{z}$ from the linear relationship $z=w x+b$, where $w$ is the layer's weight, $x$ is the input, and $b$ is an offset. Following this equation, there is an activation function $\sigma(z)$ such as a ReLu or sigmoid function depending on the neural network tasks. With the ReLu function, the DNN can avoid the gradient disappearance problem, in which parameters in some layers cannot be trained effectively owing to the increase in layers.

In our experiment, we set three layers for the DNN. All the features were inputted to the first layer, which outputted 256 internal features with the ReLu function. Then, 256 internal features were inputted to the second layer, which outputted 128 internal features with the ReLu function. Finally, 128 internal features were inputted to the third layer, which outputted the prediction.

\subsubsection{LSTM model}

The LSTM consists of several layers of cells, and sequential data are inputted to cells in the same layer. The LSTM considers sequential information in the data. Each cell has three gates: the input gate, which determines how much input information can enter the network to participate in updating the cell; the forget gate, which determines which historical information can be retained; and the output gate, which determines how much information can be outputted by the cell. LSTM can also avoid gradient disappearance with a complex model.

In our experiment, we set one layer of 5 cells for the LSTM, and each cell outputted 256 internal features. The features were inputted to a fully connected layer to output the prediction. We also bi-connected the cells because the data inputted to them depended on each other.

\subsubsection{LSTMA model}

In an LSTMA model, the attention mechanism calculates the importance of cells in the LSTM and assigns large weights to the important ones to calculate the output. The attention mechanism assigns a weight $a_{i}$ to a block of output $h_{i}$ and calculates the output $v$ with $v=\sum_{i} a_{i} h_{i}$, where $a_{i}$ is calculated with $a_{i}=\frac{\exp \left(u_{i}^{T} u_{s}\right)}{\sum_{i} \exp \left(u_{i}^{T} u_{s}\right)}$ and $u_{i}$ is calculated with $u_{i}=\tanh \left(W_{s} h_{i}+b_{s}\right)$. In these equations, $u_{s}$ and $b_{s}$ are vectors, and $W_{s}$ is a matrix. In our experiment, we set the same parameters for the LSTMA as for the LSTM. For the attention mechanism, $W_{s}$ was set to $(2 \times 256,2 \times 256)$ and $u_{s}$ to $(2 \times 256)$.

\subsubsection{Model selection and validation}


We used the root mean squared error (RMSE), mean absolute percentage error (MAPE), and coefficient of determination $\left(\mathrm{R}^{2}\right)$ to analyze the prediction results. The RMSE measures the deviation between predicted and real values, the MAPE measures the deviation between predicted and real values normalized by the predicted values, and $\mathrm{R}^{2}$ measures how well a model fits a set of data. A small RMSE and MAPE and an $\mathrm{R}^{2}$ close to 1 indicate an accurate prediction.

The DNN, LSTM, and LSTMA results are listed in Table 2. Generally, the RMSE and MAPE of the various prediction models are small and $\mathrm{R}^{2}$ is close to 1 , indicating high prediction accuracy. LSTM achieves the smallest RMSE and lowest $\mathrm{R}^{2}$, while LSTMA has the smallest MAPE. This indicates the LSTM and LSTMA models are advantageous over DNN.
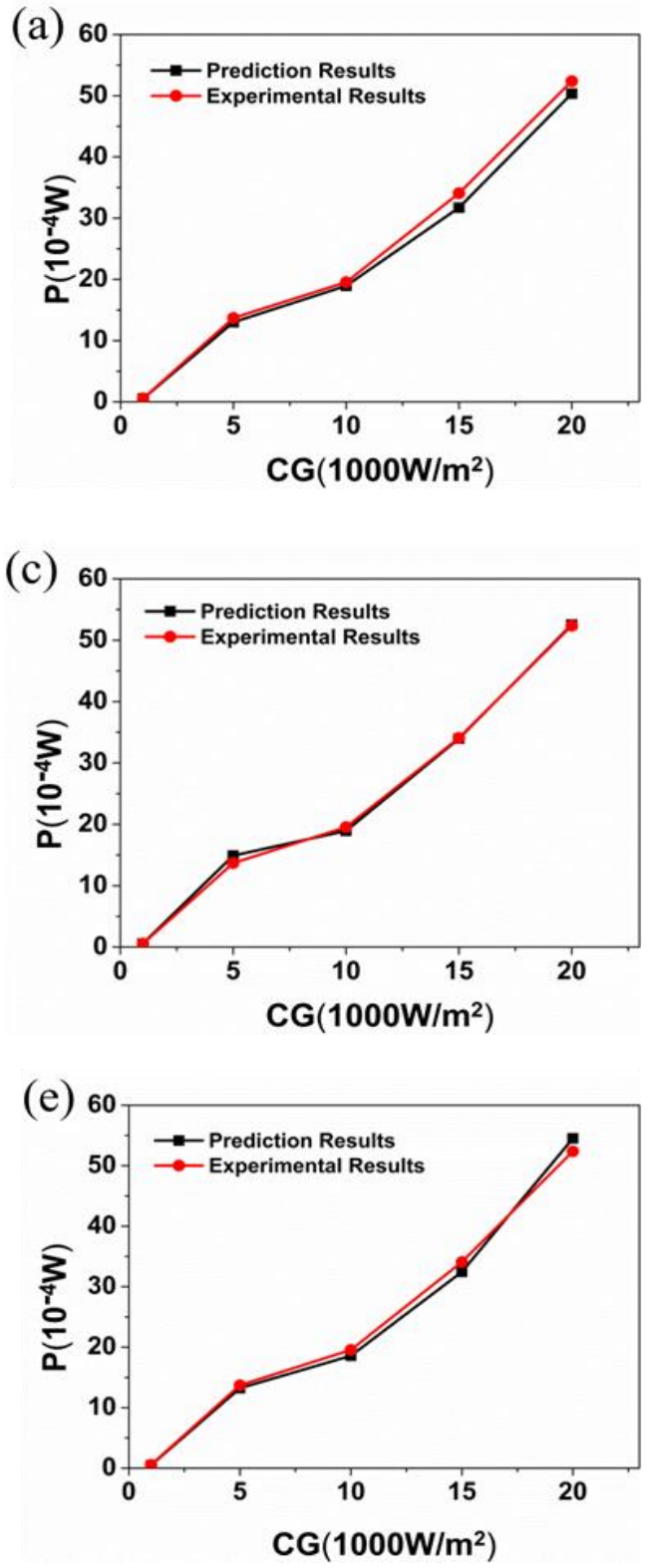

Table 2. DNN, LSTM and LSTMA model results.

\begin{tabular}{llll}
\hline Model & RMSE & MAPE & $\mathbf{R}^{2}$ \\
\hline DNN & 1.108 & 4.420 & 0.997 \\
LSTM & 0.788 & 5.239 & 0.998 \\
$\begin{array}{l}\text { LSTM } \\
\text { (Attention) }\end{array}$ & 0.990 & 4.189 & 0.997 \\
\hline
\end{tabular}

Fig. 3 shows the comparison between the model predictions and the experimental results. The relative error between the LSTM predictions and the experimental results is not more than $4 \%$, which is the best accuracy among the three models; therefore, the LSTM model should be selected as the prediction model.
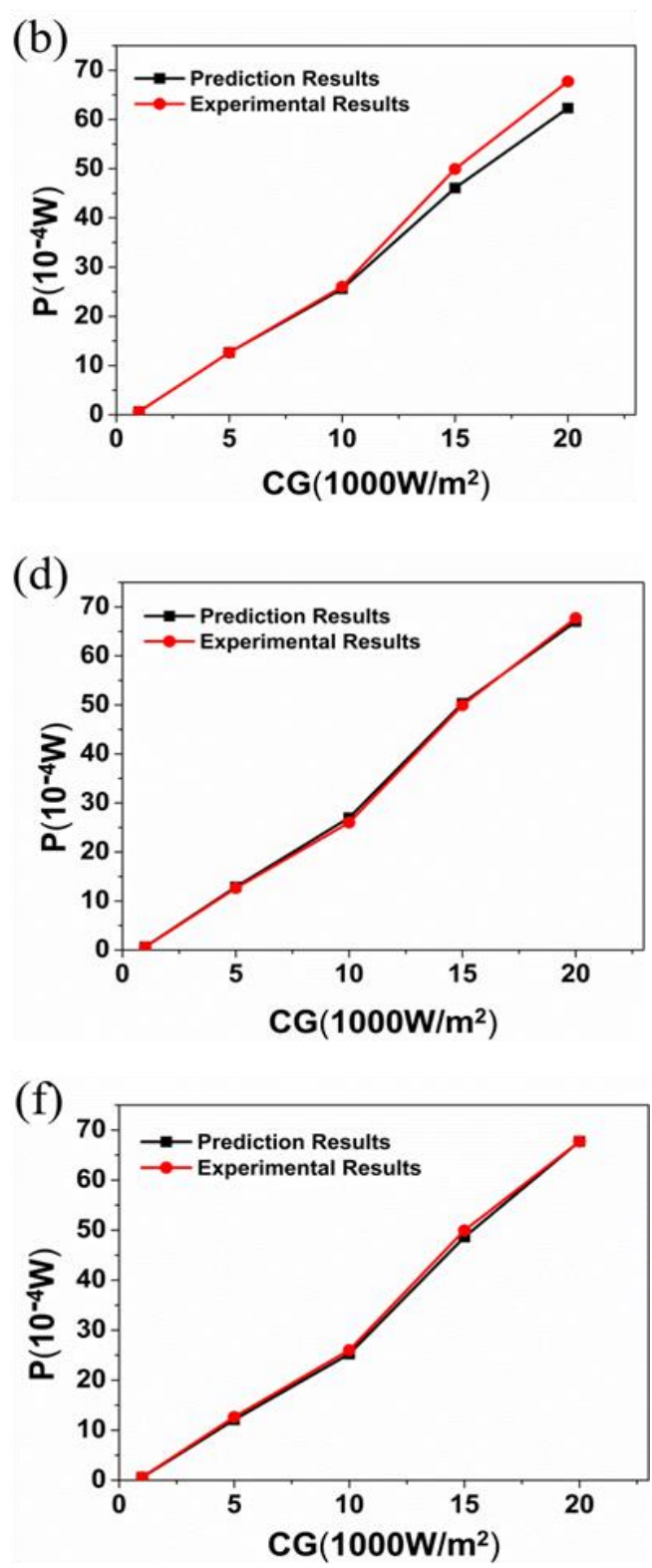

Fig. 3 Comparison between the model predictions and the experimental results of output power off TEG. Plots a and $b$ are for DNN, $\mathrm{c}$ and $\mathrm{d}$ are for LSTM, and e and f are for LSTMA. The materials are mono-crystalline silicon (a, c, and e) and gallium arsenide (b, $\mathrm{d}$, and $\mathrm{f}$ ). The external load is 3.9 , the cold-end temperature is $25^{\circ} \mathrm{C}$, and the size is $20 \times 20 \mathrm{~mm}^{2}$. 

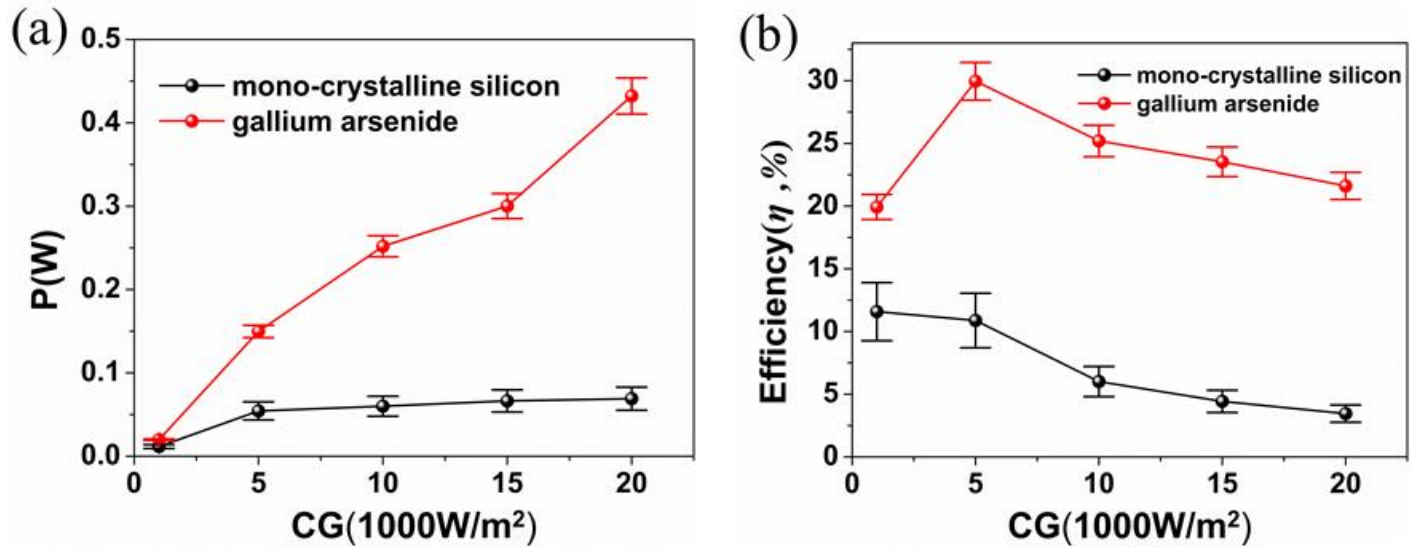

Fig. 4 Variation of (a) output power and (b) efficiency of PV module with different concentration ratios for $\mathrm{T}_{\mathrm{c}}=20{ }^{\circ} \mathrm{C}$ and $\mathrm{n}=4$.

\section{Results and Discussion}

The hybrid system performance is related to parameters such as the solar irradiation, concentration ratio, and temperatures of the hot and cold sides of the TEG. Fig. 4 shows the variation of the output power and efficiency of different PV modules with different concentration ratios. It is clear from this figure that the power output increases with increasing concentration ratio. The output power of the gallium arsenide solar cell is much higher than that of the mono-crystalline silicon cell. The output power and efficiency of the gallium
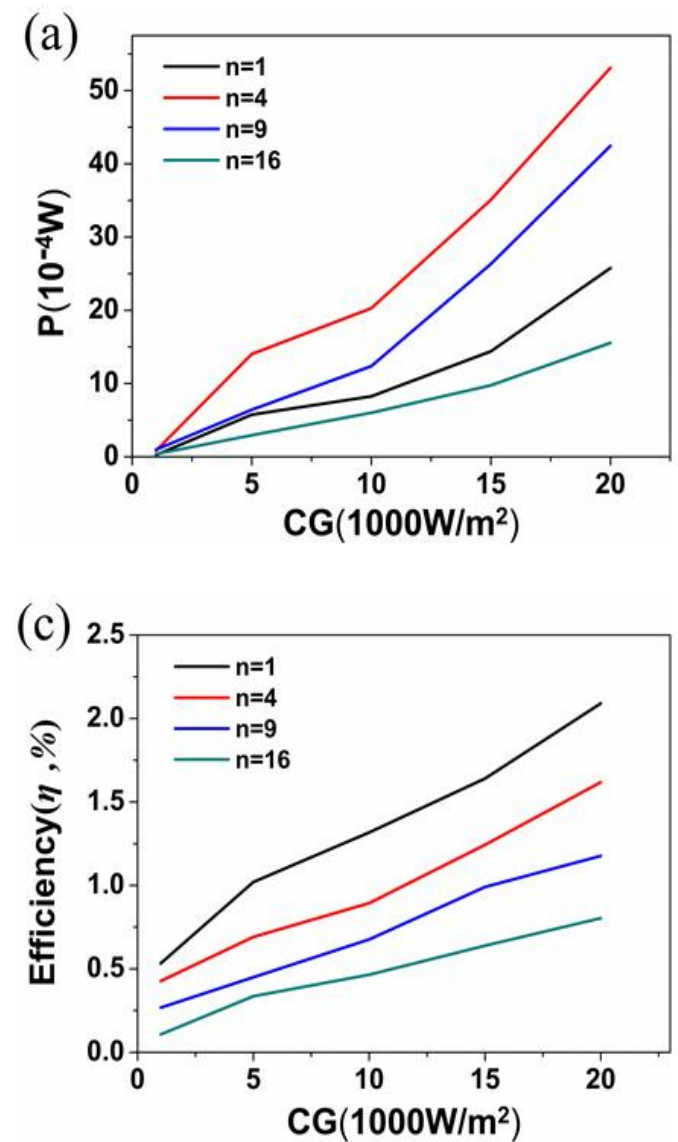

arsenide PV module are 6.25 times and 6.26 times higher than those of the mono-crystalline silicon PV module, respectively, at $\mathrm{C}=20$ and $\mathrm{T}_{\mathrm{c}}=20^{\circ} \mathrm{C}$. However, the efficiency of the gallium arsenide solar cell for $\mathrm{C}=5$ reaches a maximum and then decreases with further increase in the concentration ratio, and that of the mono-crystalline silicon cell decreases with increasing concentration ratio. Therefore, there is an optimum concentration ratio at which output power or efficiency is maximum.
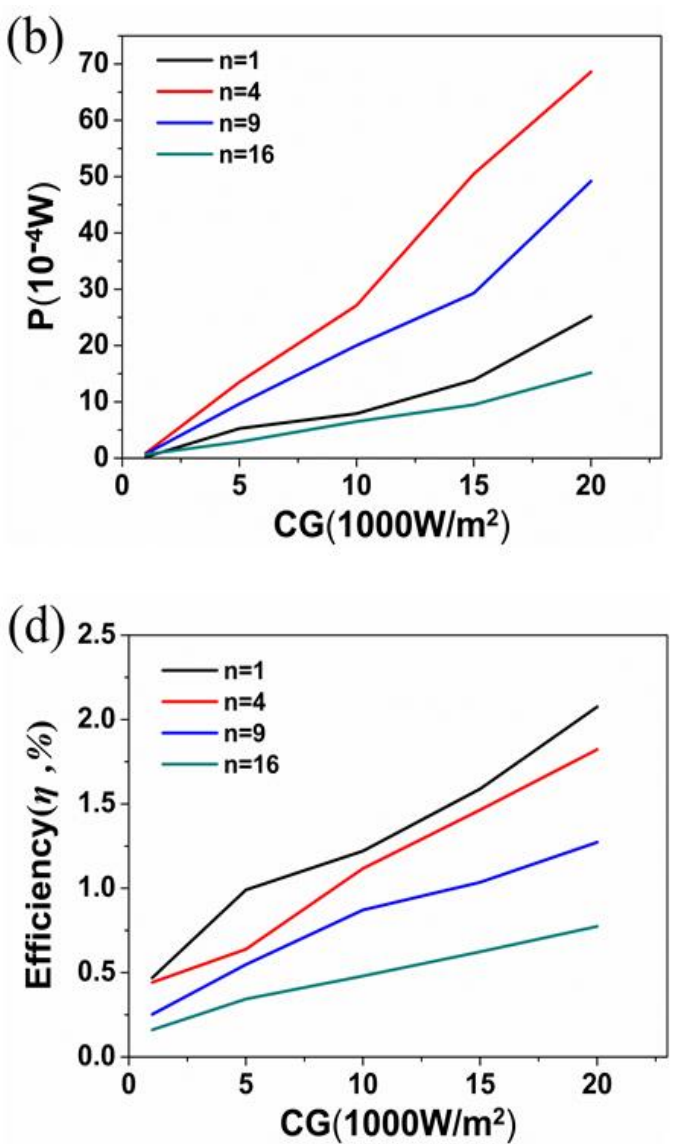

Fig. 5 Variation of output power and efficiency of TEG with concentration ratios at different values of $n$ for $\mathrm{T}_{\mathrm{c}}=20^{\circ} \mathrm{C}$. (a) and (c) are the output power and efficiency of the TEG with a mono-crystalline silicon PV module. (b) and (d) are the output power and efficiency of a TEG with a gallium arsenide PV module. 
We considered the cell area differences between the PV and TEG devices and their effect on the performance of the hybrid system. TEGs with different areas have different numbers of thermoelement pairs within them, which is one of the factors that affect output power and efficiency. In addition, when the heat transferred to the TEG through the PV solar cell is the same, a larger TEG area reduces the average temperature difference between the hot and cold sides of the TEG, which decreases the output and efficiency of the TEG in the hybrid system. Fig. 5 shows the influence of solar radiation and the value of $\mathrm{n}$ on TEG performance with $\mathrm{T}_{\mathrm{c}}=20{ }^{\circ} \mathrm{C}$. It is clear from Fig. 5 (a) and (b) that the output power of the TEG first increases and then decreases with increasing $\mathrm{n}$, but always increases with increasing solar radiation. The output power and efficiency of the TEG increase with increasing concentration ratio because more of the incident solar radiation is converted into heat, which increases the temperature of the hot side of the TEG. However, increasing the size of the TEG module decreases the temperature difference between its hot and cold sides caused by the heat conduction through the PV module, which is the main cause of the decline in output power and efficiency.

The analysis above shows that an increase in number of thermoelement pairs and decrease in temperature difference between the hot and cold sides of the TEG caused by an
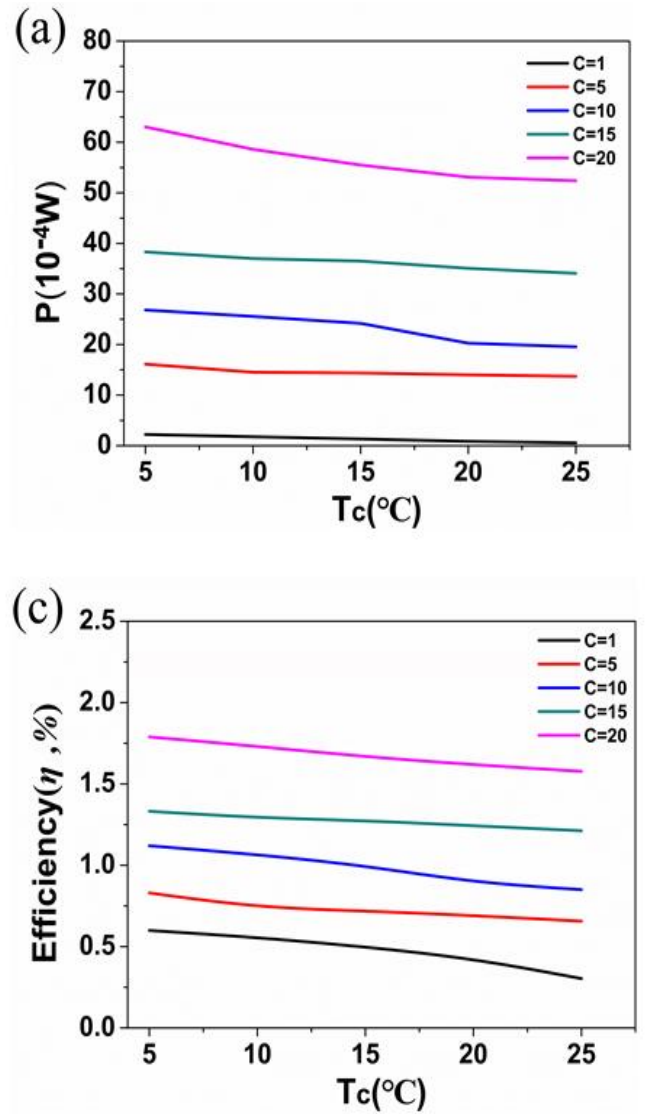

increase in TEG area both affect the TEG performance. This suggests that there is an optimal value of $n$ at which the output power or efficiency is maximum, which makes the TEG area a significant parameter for optimizing the output power and efficiency of a PV and TEG hybrid system.

Fig. 6 shows the variation in output power and efficiency with concentration ratio and cooling temperature for $n=4$. The variation in output power of the TEG with concentration ratio and $T_{c}$ is similar to that of efficiency for both the mono-crystalline silicon and gallium arsenide PV hybrid systems. The output power of the TEG decreases with increasing system cooling temperature. The reason is the same as explained earlier, that lower cooling temperature results in a larger temperature difference between the hot and cold sides of the TEG. In the mono-crystalline silicon PV hybrid system, the output power and efficiency of the TEG at $\mathrm{T}_{\mathrm{c}}=5{ }^{\circ} \mathrm{C}$ are 1.2 times and 1.13 times higher, respectively, than at $\mathrm{T}_{\mathrm{c}}=25^{\circ} \mathrm{C}$, and correspondingly 1.11 times and 1.17 times higher in the gallium arsenide PV system. The output power and efficiency of the TEG with the gallium arsenide PV system at $\mathrm{T}_{\mathrm{c}}=5{ }^{\circ} \mathrm{C}$ are 1.19 times and 1.16 times higher than those of the mono-crystalline silicon PV hybrid system. It is obvious that a gallium arsenide solar cell in a hybrid system can achieve greater output power and efficiency than those of mono-crystalline silicon.
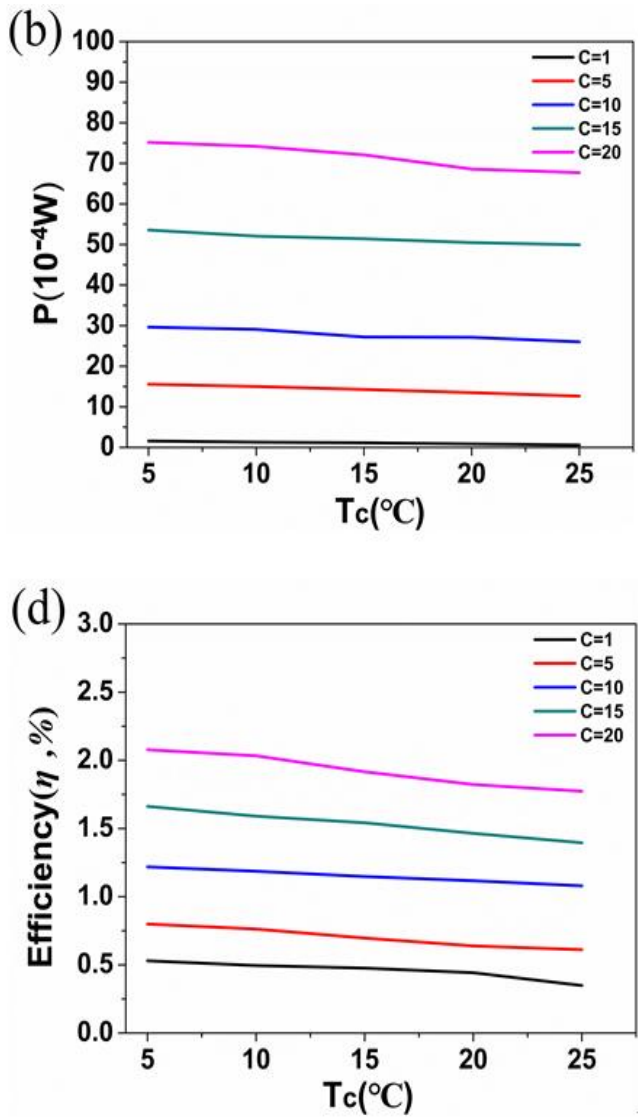

Fig. 6 Variation of output power and efficiency of TEG with cooling temperature at different concentration ratios for $n=4$. (a) and (c) are the output power and efficiency of the TEG with the mono-crystalline silicon PV module. (b) and (d) are the output power and efficiency of the TEG with the gallium arsenide PV module. 

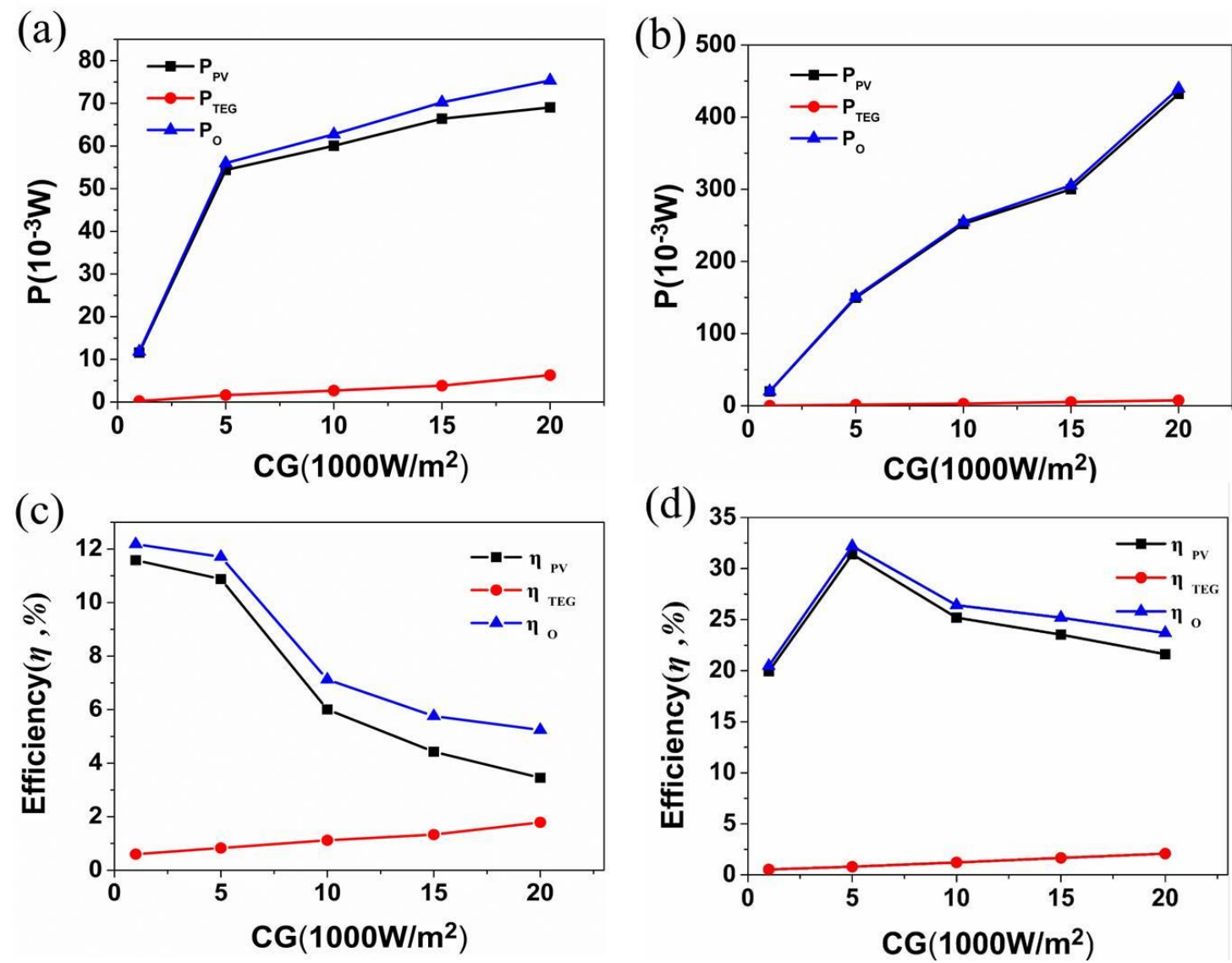

Fig. 7 Comparison of output power and efficiency of TEG, PV and hybrid system as functions with different concentration ratios for $\mathrm{T}_{\mathrm{c}}=5{ }^{\circ} \mathrm{C}$ and $\mathrm{n}=4$. (a) and (c) are the output power and efficiency of hybrid system which the TEG with the mono-crystalline silicon PV module. (b) and (d) are the output power and efficiency of hybrid system which the TEG with the gallium arsenide PV module.

We plot the efficiencies and output powers of the PV module, TEG, and hybrid system as functions of the concentration ratio in Fig. 7 , where $\mathrm{n}=4$ and $\mathrm{T}_{\mathrm{c}}=5{ }^{\circ} \mathrm{C}$. However, the electricity generated by the hybrid system is slightly higher than that by the PV owing to the additional contribution of the TEG to the whole generation power. However, the operating temperature of the PV is lower at $n=4$ than at $n=1$ because the larger TEG area at $n=4$ dissipates more heat. This is a factor that makes the total conversion efficiency of the hybrid system greater than that of a bare PV module. The contribution of the TEG power output to the overall power output of the hybrid system is much less than that of a PV, especially the gallium arsenide PV. Fig. 7(d) shows that the efficiency at the maximum power output first increases and then decreases as $C G$ is increased. This clearly shows that there is an optimum value for $C G$ at which $\eta_{\mathrm{o}}$ reaches its maximum. As a result of the trade-off between the PV and TEG performances with different TEG areas in the hybrid system, a maximum total conversion efficiency ratio of $32.2 \%$ was achieved with a TEG area of $20 \times 20 \mathrm{~mm}^{2}$ in the gallium arsenide PV hybrid system.

Fig. 8 shows the resulting optimization of the system component size using machine learning. The experimental results show that as $n$ varies, the output power of the TEG reaches a maximum under the same conditions (e.g., concentration ratio, cooling temperature) predicted by machine learning. The maximum output power of the TEG is obtained with $n=4.41$. Therefore, the system can achieve maximum output power when the ratio of the PV and TEG areas in the hybrid system is 4.41 because the TEG performance is best at this value of $n$.

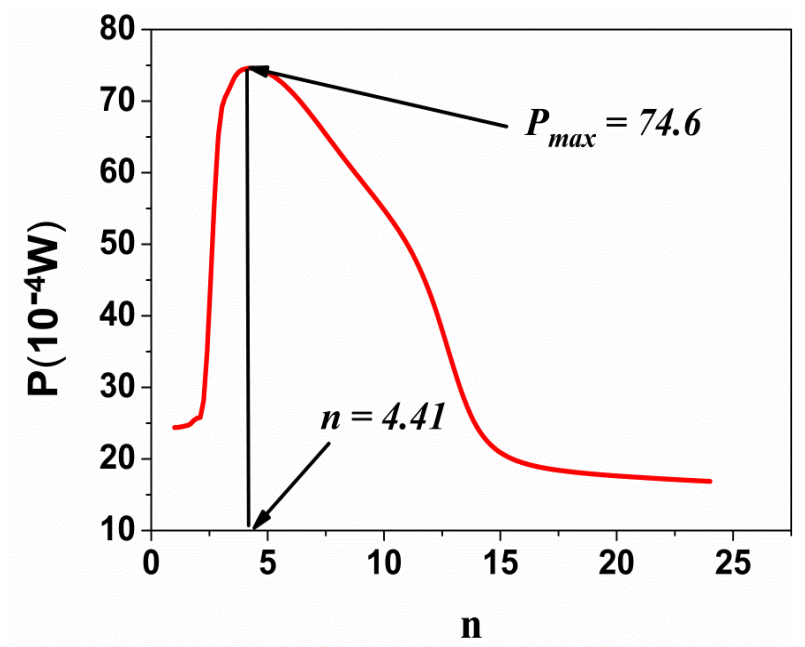

Fig. 8 Optimization of system component size based on machine learning. 


\section{Conclusion}

In this work, we analyzed the factors that affect the performance of a CPV-TEG hybrid system and used experiment and machine learning to find the structural parameters that optimize the output power of the system. The results showed that the maximum output power of this hybrid system can be obtained under the condition $\mathrm{A}_{\mathrm{TEG}} / \mathrm{A}_{\mathrm{PV}}=4.41$. Our research provides a reference for structural optimization of CPV-TEG hybrid systems.

\section{Acknowledgements}

This work was supported by the Basic Science Center Program for Ordered Energy Conversion of the National Natural Science Foundation of China (No. 51888103), the CAS Pioneer Hundred Talents Program, and the Beijing Natural Science Foundation (No. 4192044).

\section{Supporting information}

Not applicable.

\section{Conflict of interest}

There are no conflicts to declare.

\section{References}

[1] N.L. Panwar, S.C. Kaushik, and S. Kothari, Renew. Sust. Energ. Rev., 2011, 15, 1513-1524, doi: 10.1016/j.rser.2010.11.037.

[2] N. Armaroli and V. Balzani, Angew. Chem., 2007, 46, 52-66, doi: 10.1002/anie.200602373.

[3] T.T. Chow, Appl. Energ., 2010, 87, 365-379, doi: 10.1016/j.apenergy.2009.06.037.

[4] H. L. Zhang, J. Baeyens, J. Degrève, and G. Cacères, Renew. Sust. Energ. Rev., 2013, 22, 466-481, doi: 10.1016/j.rser.2013.01.032.

[5] Z.S. Xu, M.M. Meyers, B.G. Sammakia, B.T. Murray, J. Electron. Packag., 2014, 136, 041004, doi: 10.1115/1.4028060.

[6] Z. Jiang, R. Li, S.C. Zhang, and W. Liu, Phys. Rev. B, 2005, 72, doi: 10.1103/PhysRevB.72.045201.

[7] L.C. Hirst, and N.J. Ekins-Daukes, Fundamental losses in solar cells, Prog. Photovoltaics, 2011, 19, 286-293, doi: 10.1002/pip.1024.

[8] W. Lai, Y.Q. Ma, L. Zhuang, and W.M. Liu, Phys. Rev. Lett., 2019, 122, 223202, doi: 10.1103/PhysRevLett.122.223202.

[9] L. Zhang, L.F. Liu, and W.M. Liu, Sci. Rep-UK., 2013, 3, 2908, doi: 10.1038/srep02908.

[10] E. Skoplaki and J.A. Palyvos, Sol. Energy, 2009, 83, 614-624, doi: 10.1016/j.solener.2008.10.008.

[11] Y.X. Zhen, M. Yang, H. Zhang, G.-S. Fu, J.L. Wang, S.F. Wang, and R.N. Wang, Sci. Bull., 2017, 62, 1530-1537, doi: 10.1016/j.scib.2017.10.022.

[12] L. Tayebi, Z. Zamanipour, and D. Vashaee, Renew. Energ., 2014, 69, 166-173, doi: 10.1016/j.renene.2014.02.055.

[13] Y. Deng, W. Zhu, Y. Wang, and Y. Shi, Sol. Energy, 2013 88, 182-191, doi: 10.1016/j.solener.2012.12.002.

[14] A. Pereira, T. Caroff, G. Lorin, T. Baffie, K. Romanjek, S.
Vesin, K. Kusiaku, H. Duchemin, V. Salvador, N. Miloud-Ali, L. Aixala, and J. Simon, Energy, 2015, 84, 485-492, doi: 10.1016/j.energy.2015.03.053.

[15] Y.X. Zhen, M. Yang, and R.N. Wang, Front. Phys-Beijing, 2018, 14, doi: 10.1007/s11467-018-0865-0.

[16] Z. He, M.X. Foo, D. Yong, T. Ma, Y. Hao, H. Zhang, and D. Ding, ES Energy Environ., 2019, doi: 10.30919/esee8c335.

[17] Y.H. Chen, H.S. Tao, D.X. Yao, and W.M. Liu, Phys. Rev. Lett., 2012, 108, 246402, doi: 10.1103/PhysRevLett.108.246402.

[18] Y. Li, S. Witharana, H. Cao, M. Lasfargues, Y. Huang, and Y. Ding, Particuology, 2014, 15, 39-44, doi: 10.1016/j.partic.2013.08.003.

[19] W. G. J. H. M. v. Sark, Appl. Energ., 2011, 88, 2785-2790, doi: 10.1016/j.apenergy.2011.02.008.

[20] R. Lamba and S.C. Kaushik, Energ. Convers. Manage., 2016, 115, 288-298, doi: 10.1016/j.enconman.2016.02.061.

[21] S. Mahmoudinezhad, A. Rezania, and L. A. Rosendahl, Energ. Convers. Manage., 2018, 164, 443-452, doi: 10.1016/j.enconman.2018.03.025.

[22] S. Mahmoudinezhad, S.W. Qing, A. Rezaniakolaei, L. A. Rosendahl, Energ. Procedia, 2017, 142, 564-569.doi: 10.1016/j.egypro.2017.12.088.

[23] A. Rezania and L.A. Rosendahl, Appl. Energ., 2017, 187, 380-389, doi: 10.1016/j.apenergy.2016.11.064.

[24] S.Y. Wu, Y.C. Zhang, L. Xiao, and Z.G. Shen, J. Sust. Energ., 2017, 37, 533-548, doi: 10.1080/14786451.2017.1345906.

[25] D. Kraemer, L. Hu, A. Muto, X. Chen, G. Chen, and M. Chiesa, Appl. Phys. Lett., 2008, 92, 243503, doi: 10.1063/1.2947591.

[26] W. Zhu, Y. Deng, Y. Wang, S. Shen, and R. Gulfam, Energy, 2016, 100, 91-101, doi: 10.1016/j.energy.2016.01.055.

[27] T. Liao, B. Lin, and Z. Yang, Int. J. Therm. Sci., 2014, 77, 158-164, doi: 10.1016/j.ijthermalsci.2013.10.013.

[28] P. Baldi, P. Sadowski, and D. Whiteson, Nat. Commun., 2014, 5, doi: 10.1038/ncomms5308.

[29] L. Ti, Adv. Cond. Matter. Phys., 2020, 09, 11-19, doi: 10.12677/cmp.2020.92002.

[30] Z.H. Zheng, X.D. Lin, M. Yang, Z.-M. He, E. Bao, H. Zhang, and Z.Y. Tian, ES Energy Environ., 2020, doi: 10.30919/esee8c795.

[31] J. Zhang and Y. Xuan, Energy, 2019, 181, 387-394, doi: 10.1016/j.energy.2019.05.155.

[32] K.T. Park, S.M. Shin, A.S. Tazebay, H.-D. Um, J.Y. Jung, S.W. Jee, M.W. Oh, S.D. Park, B. Yoo, C. Yu, and J. H. Lee, Sci. Rep-UK., 2013, 3, doi: 10.1038/srep02123.

[33] C. Lertsatitthanakorn, S. Soponronnarit, J. Jamradloedluk, M. Rungsiyopas, and R. Sarachitti, J. Electron. Mater, 2013, 43, 2040-2046, doi: 10.1007/s11664-013-2945-9.

[34] B. Zhu, Z.Y. Huang, X.Y. Wang, Y. YU, N. Gao, F.Q. Zu, Scripta Mater., 2018, 146, 192-195, doi: 10.1016/j.scriptamat.2017.11.045. 


\section{Author information}

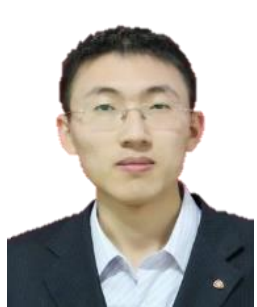

Zeming is a PHD student at the Institute of Engineering Thermophysics, Chinese Academy of Sciences, China. His research focuses on energy conversion, concentrated solar power and thermoelectric devices applications.

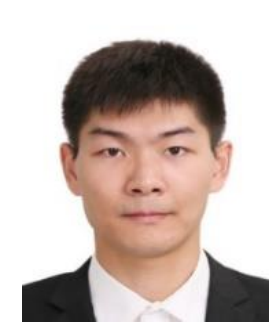

Ming Yang is an associate professor at the Institute of Engineering Thermophysics, Chinese Academy of Sciences, China. His research focuses on micro-scale heat transfer, concentrated solar power applications and topological materials. He graduated from Institute of Physics, Chinese Academy of Sciences and obtained PhD degree in theoretical physics in 2015. In 2018-2019, he was a visiting scholar in University of Colorado at Boulder.

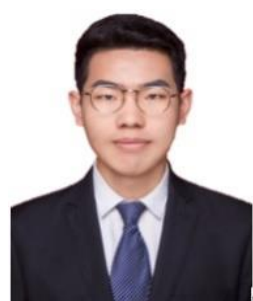

Lei Wang is a master in School of Software Engineering, Beijing Jiaotong University. His research focuses on Machine learning and natural language processing.

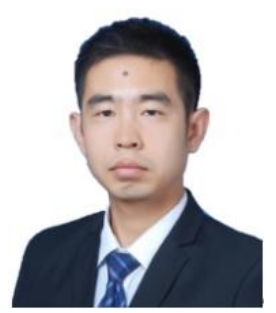

Ergude Bao is an associate professor and director of Group of Interdisciplinary Information Sciences, School of Software Engineering, Beijing Jiaotong University. His research interest is application of algorithms and artificial intelligence in various areas, such as biology, Chinese medicine, transportation and thermal physics. He received his PhD degree in Computer Science from University of California, Riverside.

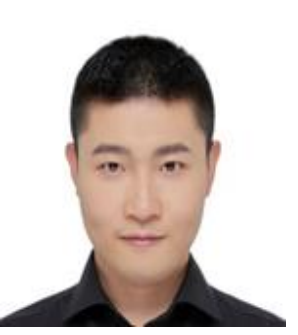

Hang Zhang is a professor in Institute of Engineering Thermophysics, Chinese Academy of Sciences and University of Chinese Academy of Sciences, in China. His research focuses on mechanisms and advanced technology on energy transport and conversions in emerging materials and equipment. He received his PhD degree in Physics from University of California, Riverside and then joined Caltech as a postdoctoral scholar.

Publisher's Note: Engineered Science Publisher remains neutral with regard to jurisdictional claims in published maps and institutional affiliation

es

